\title{
A new determination of the fine structure constant based on Bloch oscillations of ultracold atoms in a vertical optical lattice
}

\author{
Pierre Cladé, ${ }^{1}$ Estefania de Mirandes, ${ }_{1}^{1}$ Malo Cadoret, ${ }^{1}$ Saïda Guellati-Khélifa, ${ }^{2}$ \\ Catherine Schwob, ${ }^{1}$ François Nez, ${ }^{1}$ Lucile Julien, ${ }^{1}$ and François Biraben ${ }^{1}$ \\ ${ }^{1}$ Laboratoire Kastler Brossel, Ecole Normale Supérieure, \\ CNRS, UPMC, 4 place Jussieu, 75252 Paris Cedex 05, France \\ ${ }^{2}$ INM, Conservatoire National des Arts et Métiers, \\ 292 rue Saint Martin, 75141 Paris Cedex 03, France
}

\begin{abstract}
We report an accurate measurement of the recoil velocity of ${ }^{87} R b$ atoms based on Bloch oscillations in a vertical accelerated optical lattice. We transfer about 900 recoil momenta with an efficiency of $99.97 \%$ per recoil. A set of 72 measurements of the recoil velocity, each one with a relative uncertainty of about $33 \mathrm{ppb}$ in $20 \mathrm{~min}$ integration time, leads to a determination of the fine structure constant $\alpha$ with a statistical relative uncertainty of $4.4 \mathrm{ppb}$. The detailed analysis of the different systematic errors yields to a relative uncertainty of $6.7 \mathrm{ppb}$. The deduced value of $\alpha^{-1}$ is 137.03599878(91).

PACS numbers: 32.80.Pj, 32.80.Qk, 06.20.Jr, 42.65.Dr
\end{abstract}

The fine structure constant $\alpha$ plays an important role among all the physical constants because it sets the scale of electromagnetic interactions. Therefore, it can be measured in different fields of physics and so be used to test the consistency of the physics. In the Codata adjustment 1], all accurate known determinations of $\alpha$ are used to give the best estimate of $\alpha$ (labelled $\alpha_{2002}$ for 2002 adjustment). But as pointed out in [1], the actual estimate $\alpha_{2002}$ is only determined by two data and in fact mainly by the electron magnetic moment anomaly $a_{e}$ experiment. This lack of redundancy in input data is a key weakness of the Codata adjustment. For example $\alpha_{2002}$ differs from $\alpha_{1998}$ by more than one sigma mainly because of some revisions in the complicated theoretical expression of $a_{e}$ from which $\alpha$ is deduced [1]. Accurate determinations of $\alpha$ by completely different methods are absolutely needed. A competitive determination of $\alpha$ with respect to the $a_{e}$ experiment is actually the measurement of the ratio $h / m_{C s}$ (where $h$ is the Planck constant and $m_{C s}$ is the mass of the Cesium atom) using ultracold atom interferometry [2]. The fine structure constant is related to the ratio $h / m_{X}$ by $[3]$ :

$$
\alpha^{2}=\frac{2 R_{\infty}}{c} \frac{A_{r}(X)}{A_{r}(e)} \frac{h}{m_{X}}
$$

where several terms are known with a very small uncertainty: $8 \times 10^{-12}$ for the Rydberg constant $R_{\infty}[4,5]$ and $4.4 \times 10^{-10}$ for the electron relative mass $A_{r}(e)[1]$. The relative atomic mass of $\mathrm{X}$ is known with relative uncertainty less than $2.0 \times 10^{-10}$ for $\mathrm{Cs}$ and Rb atoms [ $[$ ].

In this letter, we report a new determination of the fine structure constant $\alpha$ deduced from the measurement of the ratio $h / m_{R b}$ based on Bloch oscillations. We describe a sophisticated experimental method to measure accurately the recoil velocity of a Rubidium atom when it absorbs or emits a photon. The principle of this experiment is already described in a previous paper [7]: by us- ing velocity-selective Raman transitions, we measure the variation of the atomic velocity induced by a frequencychirped standing wave. This coherent acceleration arises from a succession of stimulated Raman transitions where each Raman transition modifies the atomic momentum by $2 \hbar k$ ( $k=2 \pi / \lambda, \lambda$ is the laser wavelength), leaving the internal state unchanged. The acceleration process can also be interpreted in terms of Bloch oscillations in the fundamental energy band of an optical lattice created by the standing wave [8]: the atomic momentum evolves by steps of $2 \hbar k$, each one corresponding to a Bloch oscillation. After $N$ oscillations, we release adiabatically the optical lattice and we measure the final velocity distribution which corresponds to the initial one shifted by $2 N v_{r}$ $\left(v_{r}=\hbar k / m\right.$ is the recoil velocity). In comparison with our prior setup [7], the Bloch beams (optical lattice) and the Raman beams (velocity measurement) are now in vertical geometry (Fig @Left). This scheme is more suitable to achieve a high precision measurement of the recoil velocity, because it allows us to increase significantly the number of transferred momenta.

An atomic sample of $3 \times 10^{7}$ atoms $\left({ }^{87} \mathrm{Rb}\right)$ is produced in a magneto-optical trap (MOT), followed by a $\sigma^{+}-\sigma^{-}$ optical molasses. The final temperature of the cloud is $3 \mu \mathrm{K}$, its radius at $1 / \sqrt{e}$ is $\sim 600 \mu \mathrm{m}$ and all the atoms are in the hyperfine state $F=2$. An optical Zeeman repumper (resonant with the $F=2, F^{\prime}=2$ transition) transfers the atoms to the $F=2, m_{F}=0$ hyperfine state.

Then, a narrow velocity class is selected to $F=$ $1, m_{F}=0$ by using a counter-propagating Raman $\pi$ pulse. The non selected atoms are blown away using a resonant laser beam. After the acceleration process described later, the atomic velocity distribution is probed using a second Raman $\pi$-pulse from $\mathrm{F}=1$ to $\mathrm{F}=2$ (Fig 1 Right). The population in both levels is detected using a time of flight technique [9] . The Raman beams are produced by two stabilized laser diodes. Their beat frequency is precisely controlled by a frequency chain 


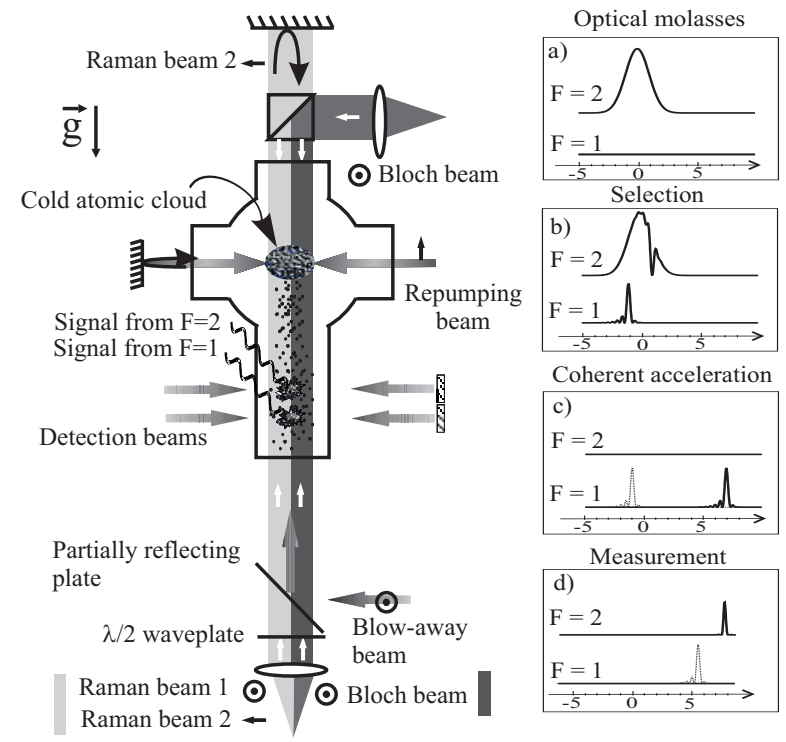

FIG. 1: Left. Experimental setup: the cold atomic cloud is produced in a MOT (the cooling laser beams are not shown), the Raman and the Bloch beams are in vertical geometry and the detection zone is at $15 \mathrm{~cm}$ below the MOT. Right. Evolution of the velocity distribution (in $v_{r}$ unit) during one experimental cycle providing one point in the final velocity distribution shown in Fig[3] (see the text and [7]).

allowing to easily switch the Raman frequency detuning from the selection $\left(\delta_{\text {sel }}\right)$ to the measurement $\left(\delta_{\text {mes }}\right)$. One of the lasers is stabilized on a highly stable FabryPerot cavity and its frequency is measured by counting the beatnote with a two-photon Rb standard [10]. The frequency of one Raman beam is linearly swept in order to compensate the Doppler shift induced by the fall of the atoms (Fig 2) (with the same slope for the selection and the measurement). The Raman beams power is $8 \mathrm{~mW}$ and their waist is $2 \mathrm{~mm}$. To reduce photon scattering and light shifts, they are blue detuned by $1 \mathrm{THz}$ from the D2 line. The duration of the $\pi$ pulse is 3.4 ms: thus, the width of the selected velocity class is $v_{r} / 50$. In order to reduce the phase noise, the Raman beams follow the same optical path: they come out from the same fiber and one of them is retroreflected (Fig 1Left).

Coherent acceleration. As shown in our previous work 7], Bloch oscillations of atoms in an optical lattice are a very efficient tool to transfer a large number of recoil momenta to the selected atoms in a short time. The optical lattice results from the interference of two counter-propagating beams generated by a Ti-Sapphire laser (waist of $2 \mathrm{~mm}$ ), whose frequency is stabilized on the same Fabry-Perot cavity used for the Raman beams and is blue detuned by $\sim 40 \mathrm{GHz}$ from the one photon transition. The optical lattice is adiabatically raised in $500 \mu \mathrm{s}$ in order to load all the atoms into the first Bloch band. To perform the coherent acceleration, the frequency difference of the two beams is swept linearly within $3 \mathrm{~ms}$

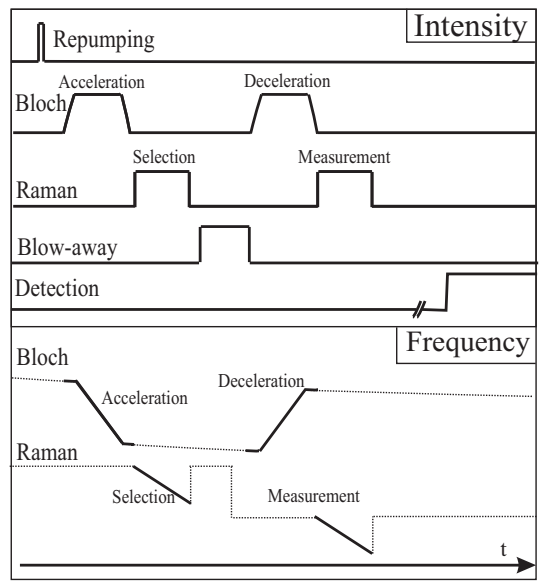

FIG. 2: Intensity and frequency timing of the different laser beams for the acceleration-deceleration sequence. (The scale of the frequency is not the same for the Bloch and the Raman beams).

using acousto-optic modulators. Then, the lattice intensity is adiabatically lowered in $500 \mu$ s to bring atoms back in a well defined momentum state. The optical potential depth is $70 E_{r}\left(E_{r}=\hbar^{2} k^{2} / 2 m\right.$ is the recoil energy). With these parameters the spontaneous emission is negligible. For an acceleration of $2000 \mathrm{~ms}^{-2}$ we transfer 900 recoil momenta in $3 \mathrm{~ms}$ with an efficiency of $99.97 \%$ per recoil. To prevent the atoms reaching the upper windows of the vacuum chamber, we use a double acceleration scheme (see Fig 2): instead of selecting atoms at rest, we first accelerate them using Bloch oscillations and then we perform the three steps sequence: selection-accelerationmeasurement. In this way the atomic velocity at the measurement step is close to zero.

In the vertical direction, an accurate determination of the recoil velocity would require a measurement of the gravity $g$. In order to get rid of gravity, we make a differential measurement by accelerating the atoms in opposite directions (up and down trajectories) keeping the same delay between the selection and the measurement $\pi$-pulses. The ratio $\hbar / m$ can then be deduced from

$$
\frac{\hbar}{m}=\frac{\left(\delta_{\text {sel }}-\delta_{\text {meas }}\right)^{u p}-\left(\delta_{\text {sel }}-\delta_{\text {meas }}\right)^{\text {down }}}{2\left(N^{u p}+N^{\text {down }}\right) k_{B}\left(k_{1}+k_{2}\right)}
$$

where $\left(\delta_{\text {meas }}-\delta_{\text {sel }}\right)^{\text {up/down }}$ corresponds respectively to the center of the final velocity distribution for the up and the down trajectories, $N^{u p / d o w n}$ are the number of Bloch oscillations in both opposite directions, $k_{B}$ is the wavevector of the Bloch beams and $k_{1}$ and $k_{2}$ are the wavevectors of the Raman beams. In Fig 3 we present two typical velocity distributions for $N^{u p}=430$ and $N^{\text {down }}=460$. The effective recoil number is then $2\left(N^{\text {up }}+N^{\text {down }}\right)=1780$. The center of each spectrum is determined with an uncertainty of $1.7 \mathrm{~Hz}\left(\sim v_{r} / 10000\right)$ for an integration time of $5 \mathrm{~min}$. 


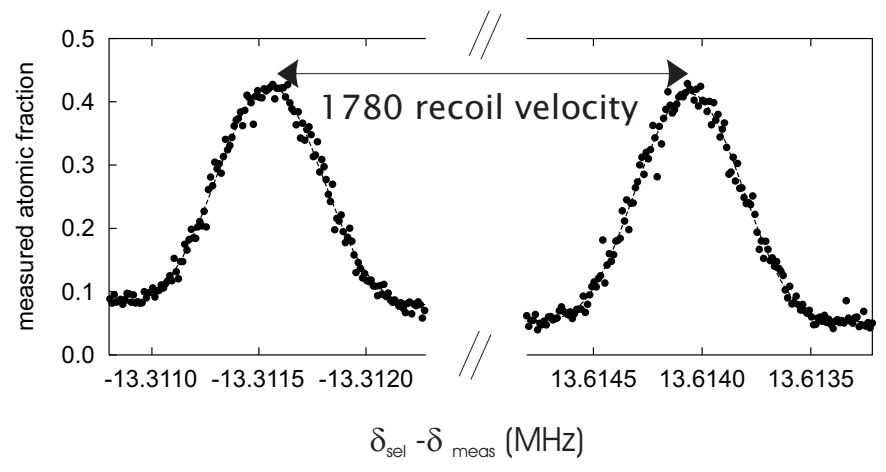

FIG. 3: Typical final velocity distribution for the up and down trajectories.

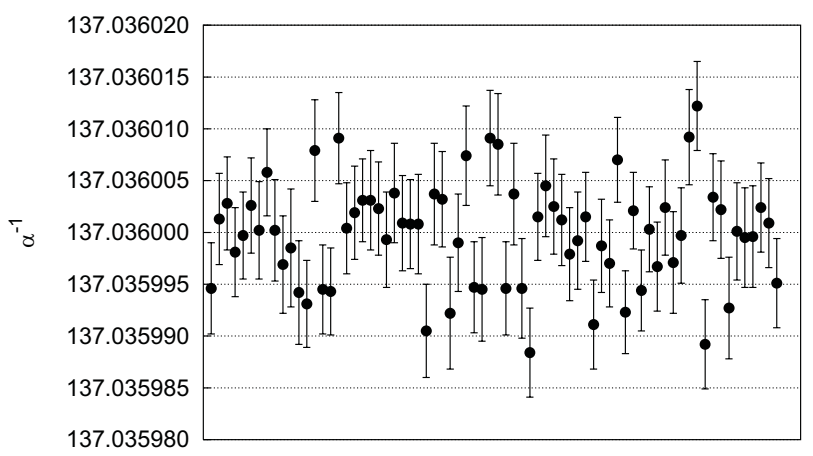

FIG. 4: Chronological display of 72 determinations of $\alpha^{-1}$.

The contribution of some systematic effects (energy levels shifts) to $\delta_{\text {sel }}$ or $\delta_{\text {meas }}$ is inverted when the directions of the Raman beams are exchanged. To improve the experimental protocol, for each trajectory, the Raman beams directions are reversed leading to the record of two velocity spectra. When the atoms follow exactly the same up or down trajectories, these systematic effects are cancelled by taking the mean value of these two measurements. Finally one determination of $\alpha$ is obtained from four velocity spectra (20 min of integration time).

The Fig 4 presents a set of 72 determinations of the fine structure constant $\alpha$. From the uncertainty of each spectrum center we deduce the standard deviation of the mean. For these $n=72$ measurements this relative uncertainty is $3.9 \mathrm{ppb}$ with $\chi^{2} \simeq 90$. Consequently, the resulting statistical relative uncertainty on $\alpha$ is $3.9 \times \sqrt{\chi^{2} /(n-1)}=4.4 \mathrm{ppb}$.

- Systematic effects analysis - We detail now all the different systematic effects taken into account to determine the final value of $\alpha^{-1}$ and its uncertainty.

Laser frequencies: The frequency of the reference Fabry-Perot cavity on which the Bloch and the Raman lasers are stabilized is checked several times during a the 20 min measurement, with respect to the Rb standard. The frequency drift is $1 \mathrm{MHz}$ and we deduce the mean laser frequency with an uncertainty smaller than $100 \mathrm{kHz}$. Thus, we assume a conservative uncertainty of $300 \mathrm{kHz}$ for the absolute determination of the different laser frequencies, which corresponds to $0.8 \mathrm{ppb}$ on $\alpha^{-1}$.

Beams alignment: We have measured the fiber-fiber coupling of the counterpropagating Bloch and Raman beams. It varies by less than $10 \%$ with respect to the maximum coupling. That corresponds to a maximum misalignment of $3.1 \times 10^{-5}$ rad between the Raman beams and of $1.6 \times 10^{-4}$ rad between the Bloch beams. The maximum systematic effect on $\alpha^{-1}$ is of $-4 \times 10^{-9}$. Thus, we correct $\alpha^{-1}$ by $(-2 \pm 2)$ ppb.

Wavefront curvature and Gouy phase: As the experimental beams are not plane waves, we have to consider the phase gradient in (2) instead of wavevectors $k$. For a Gaussian beam, the phase gradient along the propagation axis is

$$
\frac{\mathrm{d} \phi}{\mathrm{d} z}=k-\frac{2}{k w^{2}(z)}-k \frac{r^{2}}{2 R^{2}(z)} \frac{\mathrm{d} R}{\mathrm{~d} z}
$$

where $r$ is the radial distance from the propagation axis, $w(z)$ is the beam radius and $R(z)=z\left(1+\left(z_{r} / z\right)^{2}\right)$ is the curvature radius. The first corrective term (Gouy phase) originates from the spread on the transverse momenta, which is inversely proportional to the beam transverse spatial confinement. The second term comes from the spatial variation of the phase due to the curvature radius. We have measured $w(z)$ and $R(z)$ with a wavefront analyzer. The effective radial distance from the propagation axis is determined by the size of the atomic cloud $(600 \mu \mathrm{m})$ and a possible misalignment of the Bloch beam with respect to the atomic cloud. This misalignment is at maximum estimated at $500 \mu \mathrm{m}$. The correction to $\alpha^{-1}$ is $(-8.2 \pm 4) \mathrm{ppb}$. This is our dominant systematic effect.

Magnetic field: Residual magnetic field gradients contribute to the systematics in two ways. Firstly there is a second order Zeeman shift of the energy levels which induces an error in the Raman velocity measurement. Secondly, the quadratic magnetic force modifies the atomic motion between the selection and the measurement. We have precisely measured the spatial magnetic field variations using copropagating Raman transitions. The Zeeman level shift is not totally compensated by changing the direction of the Raman beams because the two up (or down) trajectories are not completely identical. They differ by about $300 \mu \mathrm{m}$, leading to a differential level shift of about $(0.3 \pm 0.1) \mathrm{Hz}$ and a $\alpha^{-1}$ correction of $(6.6 \pm 2) \mathrm{ppb}$. The magnetic force changes the atomic velocity by $(2.3 \pm 0.7) \times 10^{-6}$ recoil velocity. We correct $\alpha^{-1}$ by $(-1.3 \pm 0.4) \mathrm{ppb}$.

Gravity gradient: Gravity is not totally compensated between up and down trajectories because they differ by about $10 \mathrm{~cm}$. The correction to $\alpha^{-1}$ is $(0.18 \pm 0.02) \mathrm{ppb}$.

Light shifts: In principle, light shifts are compensated in three ways: between the selection and the measurement Raman pulses, between the upward and downward trajectories and when the Raman beams direction is changed. However this effect is not totally cancelled. 
This is firstly due to a different intensity at the selection and at the measurement because of the expansion of the cloud, secondly to spatial intensity gradient along the beams, and thirdly to intensity variations between the two Raman configurations. We calculate an effect of less than $\pm 0.2 \mathrm{ppb}$ on $\alpha^{-1}$. There is also a two-photon light shift due to the copropagating Raman beams coming out from the same fiber (before retroreflecting one of them). Its effect is larger at the measurement when Raman beams are the closest to the copropagating resonance and then corresponds to a correction on $\alpha^{-1}$ of $(-0.5 \pm 0.2) \mathrm{ppb}$.

Index of refraction: In a dispersive media of index $n$, the laser wavelength $\lambda$ becomes $\lambda / n$ and then the photon momentum transfer is $n \hbar k$. Recently, this change of the atomic recoil momentum has been observed in a KapitzaDirac interferometer 11]. In our experiment, we have measured the total ${ }^{87} \mathrm{Rb}+{ }^{85} \mathrm{Rb}$ background vapor density as $8 \times 10^{8}$ at $/ \mathrm{cm}^{3}$. The corresponding refractive index for the Bloch and Raman beams is $(n-1) \simeq-7.2 \times 10^{-10}$ and $(n-1) \simeq-3.6 \times 10^{-11}$ respectively. Thus we correct $\alpha^{-1}$ by $(-0.37 \pm 0.3) \mathrm{ppb}$. The initial density of the cold atoms is about $1 \times 10^{10}$ at $/ \mathrm{cm}^{3}$, leading to a refractive index $(n-1)_{\text {sel }} \simeq-4 \times 10^{-10}$ at the selection. The Bloch beams detuning is only $40 \mathrm{GHz}$. However, after the selection, the atomic density is lower by at least a factor 50 , thus $(n-1)_{\text {Bloch }} \simeq-2 \times 10^{-10}$. Finally for the Raman measurement $(n-1)_{\text {meas }} \simeq-10^{-12}$. We emphasize that the effect of the refractive index of the cold cloud is different than the effect of the background vapor refractive index. Especially, we have to take into account the motion of the dispersive medium (cold cloud) in the global momentum conservation and in the Doppler effect. Indeed, in the case of the Bloch beams, the accelerated cloud is itself the dispersive medium. If one has a 100\% transfer efficiency, momentum conservation seems to indicate that the refractive index of the cloud does not modify the recoil momentum transferred to the atoms. In our experiment, we have a $99.95 \%$ efficiency per Bloch oscillation, which would correspond to a modification of the atomic recoil momentum of about $5 \times 10^{-4} \times(n-1)$, leading to a negligible effect. Let us now consider the Doppler effect of an atom moving at the average velocity of the cloud, during a Raman pulse: in the frame of the cloud the length of the optical path is constant with time, so the Doppler effect is independent from the refractive index of the cloud. However, there is a small effect due to the atomic recoil. It would lead to an effect on the recoil measurement of the order of $(n-1) / N$ (where $N$ is the number of Bloch oscillations), which is also negligible. A more detailed analysis is in 12. Nerveless, we have adopted for the refractive index effect due to the cold cloud a conservative uncertainty of $3 \times 10^{-10}$ on $\alpha^{-1}$. In the table I we summarize the different systematic effects on $\alpha^{-1}$. Our determination of $\alpha^{-1}$ is $137.03599878(91)\left[6.7 \times 10^{-9}\right]$. This value is in
TABLE I: Error budget (relative uncertainty in ppb).

\begin{tabular}{lc}
\hline \hline Source & Relative uncertainty \\
\hline Laser frequencies & 0.8 \\
Beams alignment & 2 \\
Wavefront curvature and Gouy phase & 4 \\
2nd order Zeeman effect & 2 \\
Quadratic magnetic force & 0.4 \\
Gravity gradient & 0.02 \\
light shift (one photon transition) & 0.2 \\
light shift (two photon transition) & 0.2 \\
Index of refraction cold atomic cloud & 0.3 \\
Index of refraction background vapor & 0.3 \\
\hline \hline Global systematic effects & 5.0 \\
Statistical uncertainty & 4.4 \\
\hline \hline
\end{tabular}

good agreement with the two competitive determinations based on atom interferometry $\alpha^{-1}(C s)=$ $137.0360001(11)\left[7.7 \times 10^{-9}\right]$ and the $g-2$ experiment $\alpha^{-1}\left(a_{e}\right)=137.03599880(52)\left[3.8 \times 10^{-9}\right][1]$.

In conclusion, we have developed a powerful experimental approach to measure accurately the atomic recoil velocity. Thanks to the high efficiency of Bloch oscillations ( $>99.97 \%$ per recoil), we are able to transfer 900 photon momenta. To our knowledge, this is the highest number of recoils ever transferred coherently to any physical system. Our non interferometric measurement achieves a precision comparable to the best interferometric measurement [2]. An even more rigorous control of some systematics will be undertaken to reduce the uncertainty on a future determination of $\alpha$.

This experiment is supported in part by the Bureau National de Métrologie (contract 033006) and by the Région Ile de France (contract SESAME E1220). The work of E. de Mirandes is in keeping with joint Ph.D thesis between the LENS, (Universitá di Firenze) and Université Pierre et Marie Curie and is supported by European community (MEST-CT-2004-503847).

[1] P. Mohr and B.N. Taylor,Rev. Mod. Phys. 77, 1 (2005).

[2] A. Wicht et al., Physica Scripta T102, 82 (2002).

[3] B. Taylor, Metrologia. 31, 181 (1994).

[4] C. Schwob et al.,Phys. Rev. Lett. 82, 4960 (1999).

[5] Th. Udem et al., Phys. Rev. Lett. 79, 2646 (1997).

[6] M.P. Bradley et al., Phys. Rev. Lett. 83, 4510 (1999).

[7] R. Battesti et al., Phys. Rev. Lett. 92, 253001-1 (2004).

[8] M. Ben Dahan et al. Phys. Rev. Lett. 76, 4508 (1996).

[9] A. Clairon et al., IEEE Trans. Instrum. Meas. 44, 128 (1995).

[10] D. Touahri et al., Opt. Commun. 133, 471 (1997).

[11] G. K. Campbell et al., Phys. Rev. Lett 94, 170403 (2005).

[12] P. Cladé, Thesis (2005) http://tel.ccsd.cnrs.fr/tel-00010730/. 
